\title{
Barriers to implementing evidence-based practice in a private intensive care unit in the Eastern Cape
}

\author{
P Jordan, PhD; C Bowers, M Cur; D Morton, PhD \\ Department of Nursing Science, School of Clinical Care Sciences, Nelson Mandela Metropolitan University, Port Elizabeth, South Africa
}

Corresponding author: C Bowers (Candice.Bowers2@nmmu.ac.za)

\begin{abstract}
Background. Evidence-based practices (EBPs) have been promoted to enhance the delivery of patient care, reduce cost, increase patient and family satisfaction and contribute to professional development. Individual and organisational barriers can hamper the implementation of EBP, which can be detrimental to healthcare delivery.

Objective. To determine the individual and organisational implementation barriers of EBP among nurses in a private intensive care unit (ICU). Methods. A quantitative research design was used to collect data from nurses in a private ICU in the Eastern Cape Province, South Africa. The structured questionnaire (Cronbach's alpha: 0.72) was administered to 70 respondents, with a response rate of $93 \%$.

Results. Barriers at individual level were identified, and include lack of familiarity with EBP, individual perceptions that underpin clinical decision-making, lack of access to information required for EBP, inadequate sources to access evidence, inability to synthesise the literature available, and resistance to change. Barriers related to organisational support, change and operations were identified.

Conclusion. Although the findings were similar to other studies, this study showed that nurses younger than 40 years of age were more familiar with the concepts of EBP. Physicians were perceived as not being very supportive of EBP implementation. In order to enhance healthcare delivery in the ICUs, nurse managers need to take cognisance of the individual and organisational barriers that might hamper the implementation of EBP.
\end{abstract}

S Afr J Crit Care 2016;32(2):50-54. DOI:10.7196/SAJCC.2016.v32i2.253

Evidence-based practice (EBP) is increasingly being recognised in healthcare institutions as a pivotal component in patient care delivery. EBP is defined as the conscientious, explicit and judicious use of current best evidence in making decisions about the care of individual patients, and the integration of best research evidence with clinical expertise and patient values. ${ }^{[1]}$ EBP in healthcare aims to provide quality patient care by utilising the best available and valid scientific evidence. ${ }^{[2]} \mathrm{EBP}$ is achieved by accessing the best available evidence for clinical decision-making. ${ }^{[3]}$ However, other facets related to EBP should also be considered, namely clinical expertise, patient values, circumstances and the context of the working environment. ${ }^{[4]}$

Nurses are the largest group of healthcare providers globally and therefore have a key role in ensuring the promotion and delivery of quality healthcare and services. EBP can lead to enhanced quality of patient care, reduced costs, improved patient and family satisfaction, individual and professional development of nurses, enhanced organisational performance and changed outcomes. ${ }^{[5]}$ Moreover, EBP utilisation, based on international standards, enhances the quality of nursing clinical practice and promotes the best level of care for patients. ${ }^{[-7]} \mathrm{EBP}$ is important for the professional development, responsibility, and capabilities of healthcare practitioners, and many institutions are striving to integrate it into daily practice. In addition, nurses who use EBP have been shown to make better decisions in service delivery. ${ }^{[7]}$ However, there are many barriers to implementing EBP, both individual and organisational barriers.

Barriers at the level of the individual professional might include the individual's attitude, perceptions, knowledge and practices related to EBP. For instance, a study by Jordan et al. ${ }^{[8]}$ in public and private intensive care units (ICUs) in the Nelson Mandela Bay municipality, Eastern Cape Province, South Africa (SA) revealed that the majority of nurses (80 - 85\%) did not use EBP as a basis for clinical decision-making. The respondents were shown rather to base their clinical decisionmaking on tradition, what they had been taught in the unit, or on ritualistic practices. Organisational barriers might include a lack of managerial support for EBP implementation, resistance to change, unavailability of resources, and poor facilitation and support for the implementation of EBP. ${ }^{[9]}$ Additional organisational barriers include lack of time to read literature and a heavy workload. ${ }^{[6,10]}$

\section{Objective}

The study aimed to explore and describe the individual and organisational barriers to the implementation of EBP in a private ICU.

\section{Methods}

A positivistic, quantitative, exploratory research design was used for the study.

\section{Sampling and study respondents}

The research population consisted of 75 professional nurses in a private ICU in the Nelson Mandela Bay municipality, Eastern Cape, SA. Sampling was not undertaken as all professional nurses who worked in the adult ICU and who were willing to participate in the study were included. Of the 75 questionnaires distributed, 70 were returned, thus achieving a response rate of $93 \%$.

\section{Measuring instrument}

Data were collected by means of a questionnaire, which included fixed-response items. The questionnaire measured the following:

- Demographic data. A self-constructed fiveitem measurement was developed to assess the demographic profile (gender, age, years 
working in the ICU and the position held; additional qualifications) of the respondents.

- Individual barriers. A 12-item self-constructed measurement was sourced from Gerrish and Cook ${ }^{[11]}$ and Kocaman et al. ${ }^{[12]} \mathrm{A}$ fivepoint Likert scale, which ranged from (1) strongly disagree to (5) strongly agree, was used.

- Organisational barriers. An 18-item self-constructed measurement was sourced from Brown et al. ${ }^{[13]}$ and Kajermo et al. ${ }^{[14]} \mathrm{A}$ five-point Likert scale was used.

Cronbach's alpha was used to calculate the internal consistency of the measuring scale. From the statistical analysis, it can be concluded that the instrument was reliable as a score of 0.72 was obtained for the items.

\section{Ethical considerations}

Prior to data collection, ethical clearance was obtained from an institutional academic ethics committee (ethics no.: H11-HEANUR-008), and a healthcare institutional ethics committee. In addition, permission to conduct the study was obtained from the hospital and the unit managers in the ICU. Written consent from the respondents was obtained prior to commencement of the study.

\section{Data collection procedure}

The questionnaires were distributed by the researcher to the respondents at the beginning of each day and night shift and they completed the questionnaire while on duty. The participants' completed questionnaires were placed in a sealed box, and collected at a prearranged time and date from the unit manager. The data collection process continued for a week, which enabled the professional nurses on all shifts to be included in the study.

\section{Data analysis}

Statistica version 11 (StatSoft, USA) was used to analyse the data. Descriptive statistical analyses by means of frequency distributions were used to analyse the demographic data. A $\chi^{2}$ analysis, with significance set at 0.05 , was used to analyse the individual and organisational barriers. Only statistically significant results are reported on.

\section{Results \\ Demographic data of respondents}

The respondents were mostly female $(90 \%, n=63)$, between 40 and 49 years of age $(44 \%, n=31)$, with less than 10 years of work experience in ICUs $(54 \%, n=38)$ and permanently employed $(83 \%, n=58)$. Only $33 \%$ of the participants held an additional qualification in intensive care nursing with the South African Nursing Council (SANC). Table 1 presents the demographic profile of the respondents.

\section{Individual barriers to implementation of EBP}

Several individual barriers to the implementation of EBP were identified and are discussed below:

- Degree of familiarity with EBP. Of the 70 respondents, only $54 \%$ $(n=38)$ could correctly define EBP. Only $18 \%(n=12)$ were familiar with the concepts related to EBP, while $46 \%$ ( $n=32)$ incorrectly defined and understood the concept. The findings indicated that respondents younger than 40 years of age $(66 \%, n=46)$ had a better understanding of EBP compared with those older than $40(34 \%$, $n=24)$, but this was not statistically significant $\left(\chi^{2} 8.36, \mathrm{df}=3, p=0.04\right)$.

- Individual perceptions of EBP that underpin clinical decision-making. The respondents had different perceptions about what should
Table 1. Demographic profile of respondents $(N=70)$

\begin{tabular}{ll}
\hline Variable & $\boldsymbol{n}(\%)$ \\
\hline Gender & \\
Female & $63(90)$ \\
Male & $7(10)$
\end{tabular}

Age (years)

$\begin{array}{ll}<25 & 2(3) \\ 25-29 & 9(13) \\ 30-39 & 18(26) \\ 40-49 & 31(44) \\ \geq 50 & 10(14)\end{array}$

Time working in ICU (years)

$\begin{array}{ll}<1 & 5(7) \\ 1-4 & 23(33) \\ 5-9 & 10(14) \\ 10-14 & 16(23) \\ \geq 15 & 16(23)\end{array}$

Position held in ICU

Permanently employed nurses $\quad 58(83)$

Agency appointed nurses 12 (17)

Professional nurse with an additional qualification with SANC
Yes

No

underpin their clinical nursing decision-making and practices. None of the respondents relied on robust evidence to guide their clinical decision-making. Instead, the majority $(84 \%, n=59)$ relied on what was taught in their basic training, $44 \%(n=31)$ relied on traditional practices, $43 \%(n=30)$ on intuition and $39 \%(n=27)$ stated that their nursing practices were based on what was taught in the unit.

- Frequency of accessing information required for the use of implementation of EBP. The majority $(89 \%, n=62)$ of the respondents accessed evidence occasionally (once or twice a year), while $11 \%$ $(n=8)$ indicated that they accessed information on a weekly basis.

- Frequency of accessing best-practice guidelines in the ICU. Of the 70 respondents, $50 \%(n=35)$ indicated that they accessed bestpractice guidelines on an occasional basis, $14 \%(n=10)$ consulted guidelines at least weekly, $21 \%(n=14)$ accessed guidelines 1 to 3 times per month, $11 \%(n=8)$ did so 1 to 3 times a year and $4 \%(n=3)$ never accessed any best-practice guidelines.

- Information sources of evidence. On questioning the respondents on how they would access information and/or evidence, $43 \%(n=30)$ indicated that they would consult their peers, $35 \%(n=25)$ stated that they would do an unstructured internet search, $11 \%(n=8)$ would use textbooks and $11 \%(n=8)$ would consult peer-reviewed journals.

- Other sources of evidence. Of the 70 respondents, $43 \%(n=30)$ use the latest available evidence to inform their practices in the ICU, 39\% $(n=27)$ rely on what they have been taught in the unit, $17 \%(n=12)$ ask their peers and $1 \%(n=1)$ rely on ritualistic practices. Two questions were posed to assess which sources of evidence were consulted in caring for critically ill patients; $50 \%$ indicated that they would refer to information taught in the ICU to assist them in weaning a patient from a mechanical 
Table 2. Descriptive statistics: Organisational barriers related to organisational support $(N=70)$

\begin{tabular}{|c|c|c|c|c|c|c|}
\hline Statement & Mean (SD) & $\begin{array}{l}\text { Strongly } \\
\text { agree, } \\
n(\%)\end{array}$ & $\begin{array}{l}\text { Agree, } \\
n(\%)\end{array}$ & $\begin{array}{l}\text { Neutral, } \\
n(\%)\end{array}$ & $\begin{array}{l}\text { Disagree, } \\
n(\%)\end{array}$ & $\begin{array}{l}\text { Strongly } \\
\text { disagree, } \\
n(\%)\end{array}$ \\
\hline $\begin{array}{l}\text { Nurse managers are supportive of implementing } \\
\text { EBPs }\end{array}$ & $2.47(1.16)$ & $13(19)$ & $33(47)$ & $5(7)$ & $16(23)$ & $3(4)$ \\
\hline $\begin{array}{l}\text { Nursing colleagues are supportive of } \\
\text { implementation of EBPs }\end{array}$ & $2.91(1.10)$ & $6(9)$ & $24(34)$ & $13(19)$ & $24(34)$ & $3(4)$ \\
\hline Physicians are supportive of implementing EBPs & $2.99(1.11)$ & $6(9)$ & $19(27)$ & $21(30)$ & $18(26)$ & $6(9)$ \\
\hline $\begin{array}{l}\text { Nurses are open and receptive to the use of best- } \\
\text { practice guidelines }\end{array}$ & $2.54(1.03)$ & $8(11)$ & $34(49)$ & $12(17)$ & $14(20)$ & $2(2)$ \\
\hline $\begin{array}{l}\text { The organisation would be able to support best- } \\
\text { practice guideline implementation }\end{array}$ & $2.53(0.86)$ & $5(7)$ & $35(50)$ & $18(26)$ & $12(17)$ & $0(0)$ \\
\hline $\begin{array}{l}\text { The institutional management is open and willing to } \\
\text { participate in evidence uptake }\end{array}$ & $2.73(0.92)$ & $6(9)$ & $22(31)$ & $28(40)$ & $13(19)$ & $1(1)$ \\
\hline $\begin{array}{l}\text { An EBP mentor or champion is needed for the } \\
\text { implementation of EBP }\end{array}$ & $1.67(0.72)$ & $31(44)$ & $33(47)$ & $4(6)$ & $2(3)$ & $0(0)$ \\
\hline $\mathrm{SD}=$ standard deviation. & & & & & & \\
\hline
\end{tabular}

Table 3. Descriptive statistics: Organisational barriers related to organisational change $(N=70)$

\begin{tabular}{|c|c|c|c|c|c|c|}
\hline Statement & Mean (SD) & $\begin{array}{l}\text { Strongly } \\
\text { agree, } \\
n(\%)\end{array}$ & $\begin{array}{l}\text { Agree, } \\
n(\%)\end{array}$ & $\begin{array}{l}\text { Neutral, } \\
n(\%)\end{array}$ & $\begin{array}{l}\text { Disagree, } \\
n(\%)\end{array}$ & $\begin{array}{l}\text { Strongly } \\
\text { disagree, } \\
n(\%)\end{array}$ \\
\hline $\begin{array}{l}\text { I lack the authority in the critical care unit to change } \\
\text { practice to support EBPs }\end{array}$ & $2.60(1.29)$ & $16(23)$ & $24(34)$ & $7(10)$ & $18(26)$ & $5(7)$ \\
\hline $\begin{array}{l}\text { There is insufficient time to implement changes in } \\
\text { practice }\end{array}$ & $2.71(1.32)$ & $15(21)$ & $22(31)$ & $7(10)$ & $20(29)$ & $6(9)$ \\
\hline $\begin{array}{l}\text { The severity of the critically ill patient influences the } \\
\text { implementation of EBPs }\end{array}$ & $2.77(1.19)$ & $10(14)$ & $24(34)$ & 13 (19) & $18(26)$ & $5(7)$ \\
\hline $\begin{array}{l}\text { I do not feel confident about changing practice based } \\
\text { on research findings or evidence }\end{array}$ & $3.21(1.18)$ & $4(6)$ & $21(30)$ & $10(14)$ & $26(37)$ & $9(13)$ \\
\hline $\begin{array}{l}\text { There are insufficient resources to change practice to } \\
\text { support implementation of EBPs }\end{array}$ & $3.23(1.23)$ & $7(10)$ & $14(20)$ & $16(23)$ & $22(31)$ & $11(16)$ \\
\hline
\end{tabular}

ventilator, and $56 \%$ of the respondents would refer novice nurses in their units to the latest best-practice guideline when sourcing information on the prevention of ventilator-associated pneumonia.

- Inability to synthesise the amount of literature available. Many respondents $(60 \%, n=42)$ stated that the amount of information related to intensive care was too overwhelming to comprehend and synthesise, which might hamper the implementation of EBP. Furthermore, $43 \%(n=30)$ agreed that they have difficulty in critical appraisal of journal articles or guidelines.

- Resistance of nurses to change from traditional and ritualistic practices to EBP. The majority of respondents $(59 \%, n=41)$ showed resistance to change from traditional practice to EBP, while $41 \%(n=29)$ indicated that they are not resistant to change.

\section{Organisational barriers to the implementation \\ for EBP}

The organisational barriers to implementation of EBP were lack of organisational support, organisational change and operations.

- Organisational barriers related to support (Table 2). Seven items were included in this section. Two-thirds of the respondents $(66 \%, n=46)$ agreed or strongly agreed that nurse managers would support the implementation of EBP, while $27 \%(n=19)$ disagreed or strongly disagreed, and $7 \%(n=5)$ were neutral. Some respondents $(43 \%, n=30)$ agreed or strongly agreed that they would have the support of other nursing colleagues to implement EBP, while $38 \%(n=27)$ disagreed or strongly disagreed and $19 \%(n=13)$ were neutral. Physicians were perceived to be the least supportive of the implementation of EBP, as was evident by the fact that $36 \%(n=25)$ of respondents agreed or strongly agreed with that statement. A total of $60 \%$ of respondents indicated that nurses are open and receptive to the use of available best-practice guidelines, while $57 \%$ indicated that their organisation would be able to support the implementation of bestpractice guidelines in the ICU. It was noted that $39 \%(n=28)$ of the respondents indicated that the institutional management would be open and willing to participate in evidence uptake. However, the majority of the respondents (91\%) indicated that an EBP mentor or champion is needed in the ICU to aid in the effective implementation of EBP.

- Organisational barriers related to organisational change (Table 3). Five items were included in this section. The majority of the respondents $(58 \%, n=41)$ agreed that they lacked the authority to change practice in order to facilitate the implementation of EBP. 
Approximately half of the respondents $(52 \%, n=37)$ indicated that they had insufficient time to implement the change that is required for EBP, while $48(n=34)$ indicated that the illness severity of the critically ill patient hindered the implementation of EBP. Furthermore, 36\% $(n=25)$ agreed that they lacked confidence to change practice in support of EBP, 50\% $(n=35)$ disagreed or strongly disagreed and $14 \%$ $(n=10)$ were neutral. Lastly, $30 \%(n=21)$ of respondents indicated that a lack of resources was a barrier to the implementation of EBP. - Organisational barriers related to operations. Six items were included in this section. Seventy-three percent $(n=51)$ of respondents indicated that the lack of available research reports was a barrier to EBP implementation, while $66 \%(n=46)$ agreed that the lack of adequate access to computers was also a barrier. Furthermore, $44 \%$ of the respondents indicated that they have difficulty in reading and understanding research reports, and $25 \%(n=18)$ of the respondents agreed that implications of research findings for clinical practice are unclear. Other barriers that were identified by the respondents were insufficient time to implement $\operatorname{EBP}(61 \%, n=43)$, as well as an increased workload in the ICU $(56 \%, n=39)$.

\section{Discussion}

These findings highlight the fact that individual barriers might hamper the implementation of EBP in ICUs. Nearly half of the respondents showed a lack of familiarity or knowledge of EBP. Lack of knowledge of EBP among SA healthcare professionals has previously been found to be a barrier to EBP implementation. ${ }^{[15]}$ However, this is not only an SA problem as it appears that, globally, knowledge of EBP by nurses varies and in many cases is lacking. ${ }^{[7,16]}$ In this study it was found that nurses younger than 40 years of age had a better understanding of EBP, thus implying that they might be more open to the implementation of EBP. This might be due to the fact that the younger generation are more technologically inclined, thus enhancing searching strategies, or that they are more exposed to the incorporation of EBP in their curricula and teaching programmes. Relationships between the other demographic variables and EBP were not statistically significant and were not reported. The findings of the study confirm the report by Dalheim et al., ${ }^{[17]}$ who found that younger nurses tend to use EBP more than older nurses, with the latter tending to use self-experience.

Concerning individual barriers, all the respondents based their clinical decision-making primarily on traditional, ritualistic practices, on what they were taught in their basic training programme, on what was taught in the unit and on their personal intuition. A US study found that nurses in a hospital setting followed traditional practices, which were in opposition to the best evidence available. ${ }^{[18]}$ Clinical decisions that are not based on evidence could be detrimental to the care of the critically ill patient. Gerrish and Cooke ${ }^{[1]}$ found that the highest-ranked sources were tradition or ritual, intuition, information obtained from peers or colleagues and information from policy/ procedure manuals. It can be deduced that nurses in an SA context have similar tendencies in not relying on evidence to guide their decision-making.

Additional individual barriers cited were related to the frequency of accessing information and evidence, as well as the sources of retrieving such information; these were shown to be challenging to the respondents. They felt that the information found was overwhelming and difficult to synthesise, which suggests an inability to critically appraise, validate and comprehend the information in order to make a clinical decision. In a study by Baird and Miller ${ }^{[19]}$ it was found that nurses accessed evidence from research and nursing journals the least. Nurses find it a major challenge to keep updated with the literature, as there are approximately 1000 EBP publications annually, including ICU literature..$^{[12,20,21]}$

The last finding related to individual barriers was the resistance of practitioners to change from traditional practices to implementing EBP. Barker ${ }^{[22]}$ mentioned that most people are resistant to change because they are trapped in their 'comfort zones'. Williams et al. ${ }^{[23]}$ stated that often there is an organisational culture that is resistant to change and to EBP implementation. Hence, there is a need to foster an EBP culture to ensure EBP implementation. ${ }^{[24]}$

The findings also highlighted that organisational barriers could hinder the implementation of EBP in ICUs. In this study, physicians were ranked as being the least supportive of EBP implementation in the ICU, while nurse managers were the most supportive. Just over a quarter felt that nursing colleagues would be supportive of EBP implementation. In a UK study nurses ranked nursing colleagues as the most supportive regarding EBP, followed by nurse managers and then physicians. ${ }^{[11]}$ However, it is encouraging to note that nursing managers are regarded as the most supportive in this study, which is an essential factor to consider when the implementation of EBP in a private ICU is planned; this was not the case in an American study. ${ }^{[25]}$ On the other hand, the perceived lack of physician support might be detrimental to EBP implementation as physicians are one of the main stakeholders in the care of critically ill patients in private ICUs. The perceived lack of support from other non-nursing staff/management was also a major barrier to EBP implementation. ${ }^{[13]}$

Another organisational barrier to EBP implementation identified was the lack of authority to change practice. This lack of authority was identified in a review as being one of the top five barriers to the implementation of EBP. ${ }^{[23]}$ A lack of authority (13.2\%) was the greatest barrier to the implementation of EBP. ${ }^{[11]}$ In a study by Brown et al., ${ }^{[13]}$ lack of nurses' autonomy to change practice required for EBP implementation was among the top two organisational barriers.

The respondents reported a number of perceived barriers that were linked to organisational operations, including a lack of time, increased workload, a lack of readily available research reports, and a lack of access to computers. They reported that they had insufficient time to implement EBP. Lack of time has been reported elsewhere as one of the top ten barriers to EBP implementation. ${ }^{[13,26,27]}$ Lack of time is regarded as a hindrance to the implementation of EBP. Healthcare institutions tend to have a culture of 'busyness' which is valued and rewarded and which does not encourage nurses to spend time sitting and reading, but instead rewards those who visibly engage in duties with their patients. Lack of time is also linked to increased workload. ${ }^{[17]}$

Similar to these results, Williams et al. ${ }^{[23]}$ found high workload to be a major barrier to the implementation of EBP, primarily because it undermines the amount of time available for nurses to read EBP-related research. An integrative literature review by Solomons and Spross ${ }^{[6]}$ also found that workload affected EBP implementation, with 11 studies reporting that time was a major barrier.

The lack of availability of research reports was indicated as another barrier to EBP implementation. Lack of internet access at work was listed by a number of studies as being important organisational barriers to EBP implementation. ${ }^{[7,19]}$

\section{Study limitations}

The study was conducted in one private ICU in the Eastern Cape, thus limiting the generalisability of findings. However, the findings were similar to international studies cited. The questionnaire was 
self-reported and did not capture the actual practices related to EBP implementation.

\section{Conclusion}

Professional nurses in a private ICU in the Nelson Mandela Bay municipality identified individual and organisational barriers to the implementation of EBP. The study findings showed that nurses younger than 40 years are more familiar with the concepts of EBP. It is recommended that the reasons for this phenomenon be investigated in future research. In the ICU, the support of physicians for the implementation of EBP should be encouraged. In order to promote healthcare delivery and quality of patient care, nurse managers and other stakeholders in healthcare institutions should be aware of these individual and organisational barriers that might hamper the implementation of EBP.

\section{References}

1. Sackett DL, Straus SE, Richardson WS, Rosenberg W, Haynes RB. How to Practise and Teach EBM. Edinburgh: Churchill Livingstone, 2000.

2. Fortney JC, Pyne JM, Burgess JF. Population-level cost-effectiveness of implementing evidencebased practices into routine care. Health Serv Res 2014;49(6):1832-1851. http://dx.doi. org/10.1111/1475-6773.12247

3. Pearson A, Field J, Jordan Z. Evidence-based Clinical Practice in Nursing and Health Care: Assimilating Research, Experience and Expertise. Oxford: Blackwell, 2007.

4. Hoffman T, Bennett S, Del Mar C. Introduction to Evidence-based Practice. Evidence-based Practice across the Health Professions. Chatsworth: Churchill Livingstone, Elsevier, 2010.

5. Heydari A, Mazlom SR, Ranjbar H, Scurlock-Evans L. A study of Iranian nurses' and midwives' knowledge, attitudes, and implementation of evidence-based practice: The time for change has arrived. Worldviews Evid Based Nurs 2014;11(5):325-331. http://dx.doi.org/10.1111/wvn.12052

6. Solomons NM, Spross JA. Evidence-based practice barriers and facilitators from a continuous quality improvement perspective: An integrative review. J Nurs Manag 2011;19(1):109-120. http:// quality improvement perspective: An integrativer
dx.doi.org/10.1111/j.1365-2834.2010.01144.x

7. Khammarnia M, Haj Mohammadi M, Amani Z, Rezaeian S, Setoodehzadeh F. Barriers to implementation of evidence based practice in Zahedan teaching hospitals, Iran, 2014. Nurs Res Pract 2015;(2015):1-5. http://dx.doi.org/10.1155/2015/357140

8. Jordan P, van Rooyen D, Venter D. Endotracheal tube cuff pressure management in adult critical care units. S Afr J Crit Care 2012;28(1):13-16. http://dx.doi.org/10.7196/SAJCC.129

9. Squires JE, Estabrooks CA, Gustavsson P, Wallin L. Individual determinants of research Squires JE, Estabrooks CA, Gustavsson P, Wallin L. Individual determinants of research
utilization by nurses: A systematic review update. Implement Sci 2011;6(1):1-20. http://dx.doi. org/10.1186/1748-5908-6-1
10. Varaei S, Salsali M, Cheraghi MA. Implementation of evidence-based nursing practice for diabetic patients: An Iranian experience. Int J Nurs Pract 2013;19(S3):73-80. http://dx.doi.org/10.1111/ ijn. 12170

11. Gerrish K, Cooke J. Factors influencing evidence-based practice among community nurses. J Community Nurs 2013;27(4):98-101.

12. Kocaman G, Seren S, Lash AA, Kurt S, Bengu N, Yurumezoglu HA. Barriers to research utilisation by staff nurses in a university hospital. J Clin Nurs 2010;19(13-14):1908-1918. http://dx.doi. org/10.1111/j.1365-2702.2009.03032.x.

13. Brown CE, Wickline MA, Ecoff L, Glaser D. Nursing practice, knowledge, attitudes and perceived barriers to evidence-based practice at an academic medical center. J Adv Nurs 2009;65(2):371381. http://dx.doi.org/10.1111/j.1365-2648.2008.04878.x

14. Kajermo KN, Boström AM, Thompson DS, Hutchinson AM, Estabrooks CA, Wallin L. The BARRIERS scale - the barriers to research utilization scale: A systematic review. Implement Sci 2010;5(1):1. http://dx.doi.org/10.1186/1748-5908-5-32

15. McInerney P, Suleman F. Exploring knowledge, attitudes, and barriers toward the use of evidence-based practice amongst academic health care practitioners in their teaching in a South African university: A pilot study. Worldviews Evid Based Nurs 2010;7(2):90-97. http://dx.doi. org/10.1111/j.1741-6787.2009.00180.x

16. Friesen-Storms JH, Moser A, Loo S, Beurskens AJ, Bours GJ. Systematic implementation of evidence-based practice in a clinical nursing setting: A participatory action research project. I Clin Nurs 2015;24(1-2):57-68. http://dx.doi.org/10.1111/jocn.12697

17. Dalheim A, Harthug S, Nilsen RM, Nortvedt MW. Factors influencing the developmen of evidence-based practice among nurses: A self-report survey. BMC Health Serv Res 2012;2012(12):367. http://dx.doi.org/10.1186/1472-6963-12-367

18. Makic MBF, Watson CRR, Poteet AW. Examining the evidence to guide practice: Challenging practice habits. Crit Care Nurse 2014;34(2):28-45.

19. Baird LMG, Miller T. Factors influencing evidence-based practice for community nurses. Br Community Nurs 2015;20(5):233-242. http://dx.doi.org/10.12968/bjen.2015.20.5.233

20. Courtney M, McCutcheon H. Using Evidence to Guide Nursing Practice. 2nd ed. Sydney Elsevier, 2010.

21. Aveyard H, Sharp P. A Beginner's Guide to Evidence Based Practice. 2nd ed. Open University Press; 2013. http://www.mcgraw-hill.co.uk/html/0335236030.html

22. Barker J. Evidence-based Practice for Nurses. London: Sage Publications, 2010 .

23. Williams B, Perillo S, Brown T. What are the factors of organisational culture in health care settings that act as barriers to the implementation of evidence-based practice? A scoping review. Nurse Educ Today 2015;35(2):34-41. http://dx.doi.org/10.1016/j.nedt.2014.11.012

24. Stokke K, Olsen NR, Espehaug B, Nortvedt MW. Evidence based practice beliefs and implementation among nurses: A cross-sectional study. BMC Nurs 2014;13(1):8. http://dx.doi. org/10.1186/1472-6955-13-8

25. Balakas B, Sparks L, Steurer L, Bryant T. An outcome of evidence-based practice education: Sustained clinical decision-making among bedside nurses. J Pediatr Nurs 2013;28(5):479-485 http://dx.doi.org/10.1016/j.pedn.2012.08.007

26. Koehn ML, Lehman K. Nurses' perceptions of evidence-based nursing practice. J Adv Nurs 2008;62(2):209-215. http://dx.doi.org/10.1111/j.1365-2648.2007.04589.x

27. Ubbink DT, Guyatt GH, Vermeulen H. Framework of policy recommendations for implementation of evidence-based practice: A systematic scoping review. BMJ Open 2013;3(1):1-12. http://dx.doi. org/10.1136/bmjopen-2012-001881 